\title{
Function of the CaMK II on LTP of Exercise Learning
}

\author{
Xiyan Xie, Dan Xu, Shun Li, Yanfen Chen, and Lijuan Hou
}

\begin{abstract}
This $\mathrm{Ca}^{2+} /$ calmodulin-dependent kinase II (CaMKII) is an abundant synaptic signaling molecule that is essential for memory formation and the induction of synaptic potentiation. Additionally, CaMKII plays a prominent role in synaptic tagging and metaplasticity. The kinase becomes active in the presence of calcium and has the potential to switch to an autonomous mode of activity upon auto-phosphorylation. Since CaMKII can be autonomously active and required for the formation of memory, CaMKII is central to the mechanism of hippocampal, N-methyl-d-Aspartate NMDA receptor dependent long-term potentiation of synaptic transmission (LTP). However, the exact reason increased activity and how this is worked it is still matter of debate and the exact time window of increased activity and how this is terminated, There is now overwhelming evident that the expression and significance of CaMKII mRNA in hippocampus of rats whose spatial learning and memory function were enhanced by Regular Aerobic Exercise. Thus, regulation of Exercise of the expression of endogenous CaMKII may constitute important role on CaMKII signaling on LTP during memory formation.
\end{abstract}

Index Terms-Memory, LTP, exercise, camkii.

\section{INTRODUCTION}

To make and store new memories is fundamental abilities for human. Unfortunately, many diseases such as Alzheimer's disease impair memory formation. Long-term potentiation (LTP) in the CA1 region of the hippocampus has been the primary model by which to study the cellular and molecular basis of memory. It has been well documented that Calcium/calmodulin-dependent protein kinase II (CaMKII) plays a key role in plasticity of glutamatergic synaptic transmission and is known to be important for memory formation [1]-[5].

CaMKII is an abundant kinase that regulates synaptic plasticity. It is central to the regulation of glutamatergic synapses. Particularly, it is critical for the induction of LTP, a candidate mechanism of memory formation. $\mathrm{N}$-methyl-d-Aspartate (NMDA) receptors are ionotropic glutamate receptors' these coincidence detectors of synaptic activity and induce LTP by mediating a post-synaptic $\mathrm{Ca}^{2+}$ entry into the postsynaptic cell. It is believed that $\mathrm{Ca}^{2+}$ activates CaMKII through the NMDA receptor. Activated CaMKII can bind to NMDA receptor subunits and autophosphorylation at the Thr286 site of $\alpha$ CaMKII further enhances its binding affinity to $\mathrm{Ca}^{2+} / \mathrm{CaM}$ and prolongs the association of CaMKII at PSD [2-[4].

In addition to its essential role for LTP induction, CaMKII is also important for the formation of synaptic tags [6] and

Manuscript received October 17, 2012; revised December 5,2012.This work is contributed equally by Dan Xu and Xiyan Xie as co-first authors.

Lijuan Hou is with Beijing Normal University, majored in exercise physiology, Beijing China. (e-mail: houlj@bnu.edu.cn). metaplasticity. Otherwise, it is persistently activated by stimuli that elicit LTP, and can, by itself, enhance the efficacy of synaptic transmission. Thus, the CaMKII's persistent "on" state induced by LTP stimulation may allow the CaMKII autophosphorylation and dephosphorylation to serve as a memory device for information storage.

Physical exercise is a widely accepted to enhance overall health and it is well established that exercise can improve cognitive performance. It is well established that exercise regulates numerous molecular and signaling endpoints in the brain, providing multiple pathways by which exercise can modulate cognitive function and brain health. In particular, human studies have demonstrated exercise can reduce incidence of dementia attenuation of age-related loss of brain perfusion, reduced age-dependent brain atrophy [7].

We begin this review with a molecular description of CaMKII and its basic enzymatic function. We then discuss CaMKII activation occurs during LTP is necessary for memory and the important relationship between excise and memory.

\section{Biological COMPOSITION AND FUnCTION OF CAMKII}

$\mathrm{CaMKII}$ is a $\mathrm{Ca}^{2+}$-activated enzyme that is highly abundant in the brain where it constitutes $1-2 \%$ of the total protein. The kinase is enriched at synapses and is the main protein of the postsynaptic density (PSD).

CaMKII is a multifunctional serine/threonine kinas with a broad range of substrates. In mammalian cells CaMKII that is expressed from a family of four of 28 similar isoforms. They are derived from genes: $\alpha, \beta, \gamma$ and $\delta$, each of which produces mRNA that can be alternatively spliced, giving rise to at least 30 different proteins [8]. Each isoform consists of a catalytic domain, an autoinhibitory domain, a variable segment and a self-association domain (Fig. 1) [9]. The catalytic domain is inherently capable of catalysing the phosphotransferase reaction. $\alpha$ and $\beta$ isoforms are especially abundant in brain, constituting as much as $2 \%$ of total protein in the hippocampus whereas $\gamma$, and $\delta$ isoforms are ubiquitously expressed [10].

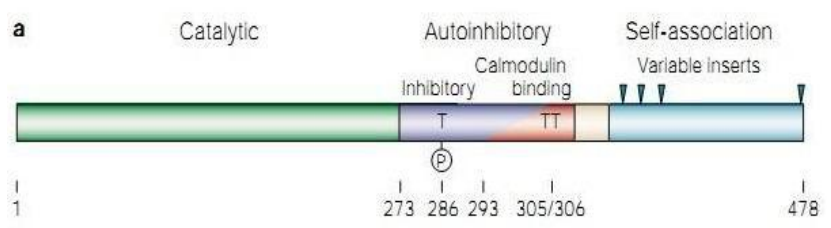

Fig. 1. The different functional domains in the primary structure of calcium/calmodulin-dependent protein kinase II (CaMKII). Each isoform consists of a catalytic domain, an autoinhibitory domain, a variable segment and a self-association domain. T represents threonine residues that are crucial phosphorylation sites.

A CaMKII constitutes the major postsynaptic density 
(PSD) protein and this isoform has been studied extensively in relation to memory formation. In recent years, advances in genetic manipulation have allowed CaMKII knockout experiments. Mice lacking $\alpha$ CaMKII show numerous deficiencies in learning and neuronal plasticity $[11,12]$, including blockage of hippocampal LTP and cortical experience-dependent plasticity.

Little is known about the function of $\beta C$ aMKII in the brain and virtually nothing about the $\gamma$ and $\delta$ isoforms [13]. $\beta C a M K I I$ is primarily neuronal, although the $\beta \mathrm{M}$ splice variant is found in skeletal muscle. The $\gamma$ and $\delta$ isoforms are expressed throughout the body and important in smooth muscle.

Autophosphorylation is the main features of CaMKII. Each CaMKII protein is composed of an N-terminal catalytic domain, an auto-inhibitory regulatory domain, and an association domain with four variable regions inter-dispersed. CaMKII inactivation state when lack of $\mathrm{Ca}^{2+} / \mathrm{CaM}$ that CaMKII inhibition area and catalytic structure combining domain curb the substrate protein and the catalytic combination. The molecular conformation changed when $\mathrm{Ca}^{2+} / \mathrm{CaM}$ combination, each catalytic structure domain close to inhibition of the structure domain phosphor late itself at Ser, Thr sites. CaMKII becomes activation and phosphor-late other target protein in cells thus appears $\mathrm{Ca}^{2+} / \mathrm{CaM}$ biological activity [1].

The second role of Autophosphorylation is promoting translocation of CaMKII to postsynaptic densities (PSDs), cytoskeletal scaffolds for the neurotransmitter receptor, ion channels, and their regulators. The target Thr286auto-phosphorylated CaMKII to the PSD initially identified a $190-\mathrm{kDa}$ binding activity, corresponding in size to the highly PSD-enriched NR2A and NR2B subunits of the NMDA receptor as a CaMKII-NMDA receptor complex from PSDs.

Recent studies have also shown that repeated electroconvulsive shock, an effective therapy for depression patients, disrupts CaMKII-GluN2B binding by causing phosphorylation of the latter at Ser1303, showing a modulation of glutamatergic transmission in depression like disorders and suggesting a reduction of GluN2B-Ser1303 phosphorylation in depression [14].

\section{FUNCTION OF CAMK II IN LEARNING-MEMORY}

The first study of the role of CaMKII in learning and memory was made in $\alpha$-CaMKII knockout mice. These mice were deficient in LTP and hippocampus-dependent spatial learning tasks [15]. CA1 hippocampal synapses have served as a model system by which to understand synaptic plasticity. The study of LTP has been the demonstration that NMDA receptor can act as a detector of coincident activity in the preand postsynaptic cells. The channels formed by these receptors open efficiently only when glutamate is released from the presynaptic terminal and the postsynaptic cell is strongly depolarized. Channel opening produces a rise in $\mathrm{Ca}^{2+}$.

CaMKII is a family of ubiquitous with broad substrate specificity and regulates many physiological responses to $\mathrm{Ca}^{2+}$ mobilization [16].It is major downstream effectors of
$\mathrm{Ca}^{2+}$ signaling in eukaryotic cells. The passage of glutamate receptor coupling with $\mathrm{Ca}^{2+}$ open and $\mathrm{Ca}^{2+}$ into the cell that increased $\mathrm{Ca}^{2+}$ concentration in cells after nerve impulses caused glutamate receptor activated. A rise in intracellular $\mathrm{Ca}^{2+}$ concentration leads to binding of $\mathrm{Ca}^{2+}$ to calmodulin (CaM), which binds to and activates CaMKII. Upon activation, CaMKII becomes auto-phosphorylated at Thr286. This autophosphorylation mechanism of $\alpha$ CaMKII initiated by calcium influx into the postsynaptic cell prevents $\alpha$ CaMKII from reverting to its resting state even after calcium levels drop to baseline levels. It is this switch to a calcium independent state which allows signals to be potentiated for longer within the cell [2], [17].

CaMKII can bind to a wide variety of proteins in the postsynaptic density (PSD). These proteins include NMDA receptor subunits $\alpha$-actinic 1 and 4, Densin-180, SynGap- $\beta$, Synapsin 1, Connexin36, L-Type $\mathrm{Ca}^{2+}$ channels. All NMDA receptor subunits have been shown to interact with CaMKII, although the results seem to be strongly dependent on the assay conditions and the phosphorylation status of CaMKII. This unique mechanism has been shown to play a special role in LTP formation, but not necessarily LTP storage [3, 18].

Without $\mathrm{Ca}^{2+}$ in vitro testing CaMKII activity, Fukunaga found that CaMKII activity increases after induction LTP in the slices of hippocampal and the active increase state could be lasting more than an hour[19].

It is not affect the synaptic transmission and activity of $\mathrm{Ca}^{2+} / \mathrm{CaM}$ dependent kinases, but the induction of LTP out of control which mediated by NMDA receptors in CA1 area of hippocampus and emerge space learning disabilities when Knockout $\alpha$ CaMKII or gene mutations $\alpha$ CaMKII of normal mice[20].

The function of CaMKII in synaptic plasticity has been most extensively studied at excitatory synapses in adult hippocampal area CA1 [21]. In the absence of T286 autophosphorylation there is a complete block of NMDA receptor dependent LTP [22]. Behavioral studies with $\alpha$ CaMKII autophosphorylation-deficient mutant mice have shown that the T286 autophosphorylation is essential for induction of transcription during memory consolidation [23]. Recent studies in Lymnea suggest that intrinsic activation of CaMKII contributes to late memory consolidation [24].

\section{EFFECT OF EXERCISE ON CAMKII IN LEARNING-MEMORY}

Physical exercise is a widely accepted behavioural strategy to enhance overall health. Human and animal studies demonstrate that exercise participation is a powerful behavioral intervention to improve cognitive function and brain health. In particular, human studies have demonstrated robust effects of exercise in the aged population, where higher physical activity is associated with improved cognitive scores on multiple aspects of cognition including executive function as well as with reduced incidence of dementia [25]. Rodent studies demonstrate that exercise can facilitate acquisition and/or retention in various hippocampal-dependent tasks including the Morris water maze, radial arm water maze and object recognition [26].

Alzheimer's disease (AD) is a neurodegenerative disorder and Genetic factors are known to play a role in $\mathrm{AD}$ 
vulnerability, yet less than $1 \%$ of incident $\mathrm{AD}$ cases are directly linked to genetic causes, suggesting that environmental variables likely play a role in the majority of cases. Several recent human and animal studies have examined the effects of behavioural factors, specifically exercise and psychological stress, on AD vulnerability. Some human studies suggest that psychological stress can increase the risk of developing $\mathrm{AD}$, while other studies suggest that exercise can significantly reduce AD risk through multiple intracellular pathways, including cAMP response element binding protein (CREB), CaMKII, and mito-gen-activated protein kinase (MAPK) [27].

Regular and moderate exercise has been considered an interesting neuroprotective strategy [27] while there is controversial evidence showing brain mitochondrial dysfunction, oxidative damage and decreased neurotrophin levels after high-intensity exercise, which presumably worsens cognitive performance [28].

Long-term regular appropriate exercise could improve spatial learning-memory of rats and increased the expression of PSD-95, CaMKII, Synapsin signal transduction Pathway [29]. Other investigation found that the expression of CaMKII mRNA increased significantly in hippocampus of rats whose spatial learning and memory function were enhanced by regular aerobic exercise and proved CaMKII plays an important role in Learning-Memory [30].

\section{CONCLUSION}

A large number of studies show the role of CaMKII in LTP. There is thus little doubt that CaMKII is activated during LTP induction which is necessary and sufficient for LTP. It is also clear that CaMKII can strengthen synaptic transmission by multiple mechanisms. The recently discovered binding of CaMKII to NMDA receptors in the PSD could be an initial step in this structural process. PSD-specific receptor and scaffolding proteins, such as NR2B and densin-180, are attractive candidates for this role. However, further work is needed in order to understand the detailed mechanism of CaMKII translocation and high affinity PSD binding.

Several recent studies have proved that exercise plays an important role in Learning-Memory. Robust effects of exercise are good for aged population, $\mathrm{AD}$ and so on. CaMKII is central to the mechanism of hippocampal, NMDA receptor-dependent LTP. Also, CaMKII can be autonomously active and required for the formation of memory. Although the mechanisms by which physical exercise alters brain function are not really clear, it appears that neuroprotective properties of exercise could be related to synaptic remodeling. Lifestyle is an important factor which affects the level of the residents' health. Maybe exercise not only keeps the residents' health but improve Learning-Memory through CaMKII signaling on LTP during memory formation.

\section{ACKNOWLEDGMENT}

This research was supported by the Fundamental Research Funds for the Central Universities, China.

\section{REFERENCES}

[1] W. Lucchesi, K. Mizuno, and K. P. Giese, "Novel insights into CaMKII function and regulation during memory formation," Brain Research Bulletin, vol. 85, no.1, pp. 2-8, 2011.

[2] K. P. Giese, N. B. Fedorov, R. K. Filipkowski, and A. J. Silva, "Autophosphorylation at Thr286 of the alpha calcium-calmodulin kinase II in LTP and learning," Science, vol. 279, no. 5352, pp. 870-873, 1998.

[3] S. Strack, R. B. McNeill, and R. J. Colbran, "Mechanism and Regulation of Calcium/Calmodulin-dependent Protein Kinase II Targeting to the NR2B Subunit of the N-Methyl-D-aspartate Receptor," Biol. Chem. vol. 275, no. 31, pp. 23798-23806, 2000.

[4] J. Steven and K. U. Bayer, " $\mathrm{Ca}^{2+} /$ Calmodulin-Dependent Protein Kinase II (CaMKII)," Protein Kinase Technologies, Neuro methods, vol. 66 , no. 221 , pp. 50-51, 2012

[5] R. J. Colbran and A. M. Brown, "Calcium/calmodulin-dependent protein kinaseII and synaptic plasticity," Curr. Opin. Neurobiol, vol. 14, no. 3, pp. 318-327, 2004

[6] K. Shen and M. N. Teruell, "Molecular memory by reversible translocation of calcium/calmodulin dependent protein kinase II," Nature neuroscience, vol. 3, no. 9, pp. 881-885, 2000.

[7] S. J. Colcombe, K. I. Erickson, N. Raz, A. G. Webb, N. J. Cohen, and E. McAuley, "Aerobic fitness reduces brain tissue loss in aging humans," Gerontol A Biol Sci Med Sci, vol. 58, no. 2, pp. 176-180, 2003.

[8] T. R. Gaertner, S. J. Kolodziej, D. Wang, J. K. Koomen, and M. N Stoops, "Comparative analyses of the three-dimensional structures and enzymatic properties of alpha, beta, gamma and delta isoforms of $\mathrm{Ca}^{2+}$-calmodulin-dependent protein kinase II," Biol. Chem., vol. 279, no. 13 , pp. 12484-1249, 2004.

[9] J. Lisman, H. Schulman, and H. Cline, "The molecular basis of CaMKII function in synaptic and behavioural memory," Nat Rev Neurosci., vol. 3, no. 3, pp. 175-90, 2002.

[10] N. E. Erondu and M. B. Kennedy, "Regional distribution of type II $\mathrm{Ca}^{2+} /$ calmodulin-dependent protein kinase in rat brain," Neurosci., vol. 5, no. 12 , pp. $3270-327,1985$.

[11] A. J. Silva, T. W. Rosahl, P. F. Chapman, Z. Marawitz, and E. Friedman, "Impaired learning in mice with abnormal short-lived plasticity," Curr Bio, vol. 6, no. 11, pp. 1509-1518, 1996.

[12] S. Glazewski, C. M. Chen, A. Silva, and K. Fox, "Requirement for $\alpha$-CaMKII in experience-dependent plasticity of the barrel cortex," Science, vol. 272, no. 5260, pp. 421-423, 1996.

[1] W. Lucchesi, "Structure-function of the multifunctional $\mathrm{Ca}^{2+} /$ calmodulin-dependent protein kinase II," Biochem., vol. 364, no. 3, pp. 593-606, 2002.

[13] F. Fumugalli, M. Pasini, A. Sartorius, R. Scherer, G. Racagni, M. A. Riva, and P. Gass, "Repeated electroconvulsive shock (ECS) alters the phosphorylation of glutamate receptor subunits in the rat hippocampus," Int. Neuropsychopharmacol, vol. 13, no. 9, pp. 1255-1260, 2010.

[14] A. J. Silva, R. Paylor, J. M. Wehner, and S. Tonegawa, "Impaired spatial learning in $\alpha$-calcium-calmodulin kinase II mutant mice," Science, vol. 257, no. 66, pp. 206-211, 1992.

[15] A. P. Braun and H. Schulman, "A non-selective cation current activated via the multifunctional $\mathrm{Ca}^{2+}$-calmodulin-dependent protein kinase in human epithelial cells," Physiol., vol. 488, no. 1, pp. 37-445, 55.

[16] A. C. Easton, " $\alpha$ CaMKII autophosphorylation controls exploratory activity to threatening novel stimuli," Neuropharmacology, vol. 61, no, 8, pp. 1424-1431, 2011.

[17] E. E. Irvine, L. S. Hertzen, F. Platter, and K. P, Giese, "Alpha CaMKII autophosphorylation: a fast track to memory," Trends Neurosci., vol. 29, no. 8, pp. 459-465, 2006.

[18] K. Fukunaga, L. Stoppini, and E. Miyamoto, "Long-term potentiation is associated with an increased activity of $\mathrm{Ca}^{2+} /$ calmodulin-dependent protein kinase II,” Biol. Chem., vol. 268, no. 11, pp. 7863-7867. 1993.

[19] K. Fukunaga and E. Miyamoto, "Current studies on a working model of CaM kinase II in hippocampal long-term potentiation and memory," Jpn J Pharmacol, vol. 79, no. 1, pp. 7-15, 1999.

[20] Y. Elgersma, J. D. Sweatt, and K. P. Giese, "Mouse genetic approaches to investigating calcium/calmodulin dependent protein kinase II function in plasticity and cognition," Neurosci., vol. 24 , no. 39 , pp. 8410-8415, 2004.

[21] R. L. Redondo, H. Okuno, P. A. Spooner, B. G. Frenguelli, H. Bito, and R. G. Morris, "Synaptic tagging and capture: differential role of distinct calcium/calmodulin kinases in protein synthesis-dependent long-term potentiation," Neurosci, vol. 30, no.14, pp. 4981-4989, 2010.

[22] L. S. V. Hertzen and K. P. Giese, "Alpha-isoform of $\mathrm{Ca}^{2+} /$ calmodulin-dependent kinase II autophosphorylation is required 
for memory consolidation-specific transcription," Neuro report, vol. 16, no.12, pp. 1411-1414, 2005.

[23] H. Wan, B. Mackay, H. Iqbal, S. Naskar, and G. Kemenes, "Delayed intrinsic activation of an NMDA-independent CaM-kinase II in a critical time window is necessary for late consolidation of an associative memory," Neurosci, vol. 30, no. 1, pp. 56-63, 2010.

[24] E. B. Larson, L. Wang, J. D. Bowen, W. C. McCormick, and L. Teri, "Exercise is associated with reduced risk for incident dementia among persons 65 years of age and older," Ann Intern Med, vol. 144, no. 2, pp. 73-81, 2006

[25] O. F. Khabour, K. H. Alzoubi, M. A. Alomari, and M. A. Alzubi, "Changes in spatial memory and BDNF expression to concurrent dietary restriction and voluntary exercise," Hippocampus, vol. 20, no.5, pp. 637-645, 2010.

[26] V. R. N. Elsner, "Effect of different exercise protocols on histone acetyltransferases and histone deacetylases activities in rat hippocampus," Neuroscience, vol. 192, no. 29, pp. 580-587, 2011.

[27] A. S. Aguiar, "High-intensity physical exercise disrupts implicit memory in mice: involvement of the striatal glutathione antioxidant system and intracellular signaling," Neuroscience, .vol. 171, no. 4, pp. 1216-1227, 2010.

[28] B. Junwei, "Effect of Exercise on Spatial Learning and Memory Function of Rats and PSD-95, CaMK11, Synapsin I in the Brain," M. S. thesis, Dept. Electron. Eng., East China Normal University, Shanghai, China, 2007.

[29] B. Junwei, "Expression of CaMKII mRNA in the Hippocampus of the Rat as their spatial Learning and Memory were Enhanced by Regular Aerobic Exercise," Journal of Jilin Institute of Physical Education, vol. 25 , no.16, pp. 56-58, 2009.

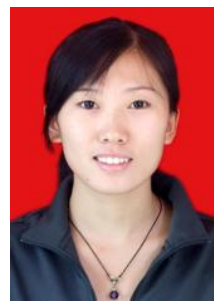

Xiyan Xie was born on 18th April, 1987, who will obtain her master's degree in 2013 from Beijing Normal University, majored in Sports health and sports nutrition. Now Xiyan Xie is a student in BNU, and learns courses on Sports health and sports nutrition. Her research interesting is focused on mechanism of sulfur dioxide causing neurological damage and the protecting of exercise. Xiyan Xie published some papers in key magazines.

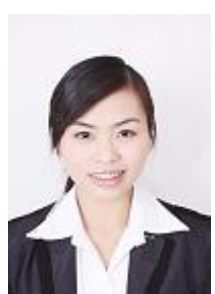

Dan Xu was born on 2th Sep, 1986, who will get master's degree in 2013 from Beijing Normal University, majored in exercise physiology. Now Dan Xu is a student in BNU, and learns courses on exercise physiology. Her research interesting is focused on central mechanism of exercise-induced fatigue and motor control. Dan Xu published some papers in key magazines.

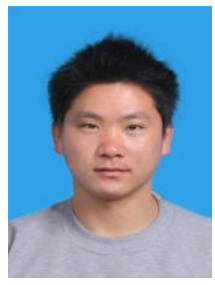

Shun Li was born on 3th Feb, 1986, who got bachelor's degree in 2012 from Beijing Normal University, majored in Athletic Training. Now Shun $\mathrm{Li}$ is a teacher in Da Tong High School Attached to Beijing Normal University, and learn courses on exercise physiology.

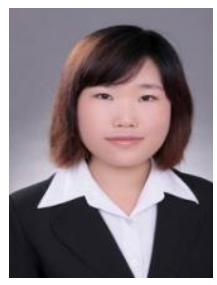

Yanfen Chen was born on 11th October, 1987, who will get master's degree in 2013 from Beijing Normal University, majored in Sports health and sports nutrition. Now Yanfen Chen is a student in BNU, and learns courses on Sports health and sports nutrition. Her research interesting is focused on study the neurotoxic effects of sulfur dioxide and the protective effect of the pre-exercise. Yanfen Chen published papers in key magazines.

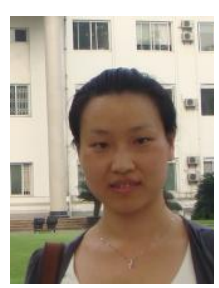

Lijuan Hou was born on 23th Oct, 1979, who got $\mathrm{PhD}$ degree in 2004 from Beijing Normal University, majored in exercise physiology. Now Lijuan is a teacher in BNU, and teach exercise physiology, biology techniques, biochemistry etc. Her research interesting is focused on central mechanism of exercise-induced fatigue and motor control. Dr. Hou published more than 10 papers in key magazines and attend some writing in related books. 